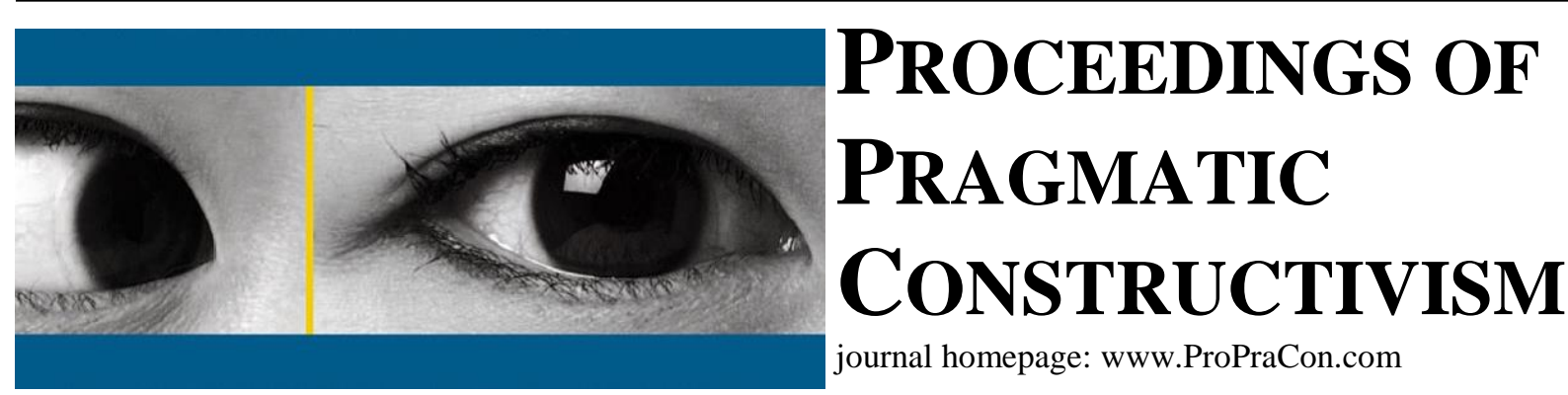

\title{
Performance Management of Cultural Events - The Case of Aarhus European Capital of Culture 2017
}

\author{
Jakob Liboriussen \\ Department of Management, \\ Arhus University, Denmark \\ E-mail: jml@mgmt.au.dk \\ Hanne Nørreklit \\ Department of Management, \\ Arhus University, Denmark \\ E-mail: hannenorreklit@mgmt.au.dk \\ Mihaela Trenca \\ Department of Management, \\ Arhus University, Denmark \\ E-mail: mtr@mgmt.au.dk
}

This paper investigates how cultural organizations can practice performance management to account for their cultural productions without interfering too much in the creative process of the artistic people. The cultural sector presents a managerial challenge for controlling and evaluating the performance of the organizations as it is characterized by high uncertainty and a need for creative productions. Dominating accounting literature offers two different methodological approaches to performance management - the principal-agent approach and the trust-based approach. Cultural organizations and managers that are using a principal-agent approach risk to suppress the creative aspects of the cultural productions because of its fundamental view on human beings as opportunistic agents and its heavy use of extrinsic based rewards, while the trust-based approach may present a controlling risk as the system may become too loose and the trust in people too naive. It is therefore of high importance for cultural organizations to find the right balance of performance management and control to avoid the different potential pitfalls of the two methodological approaches.

Focusing on the problem above, the paper presents a within-case study of the Grant Payment Team (GPT) in the Aarhus 2017 Foundation which was created in connection to the European Capital of Culture (ECoC) designation of the Danish city, Aarhus. The Foundation is responsible for the application, organization, monitoring, and reporting of the programme. The ECoC event is evaluated by RethinkIMPACTS 2017, which is a partnership between Aarhus University and Aarhus 2017 that invites researchers from various disciplines to investigate and evaluate aspects of the event (RethinkIMPACTS 2017, 2018). The Foundation established a Grant Payment Team whose responsibility it was to account for the grants given to the projects that were chosen to be a part of the ECoC event. To provide insight into 'the Aarhus 2017 way' of doing performance management, this paper takes its point of departure in this team.

Our analysis takes place on the operational level of the Foundation and shows how the employees of the GPT have used a pragmatic approach to solve their task of accounting for the cultural projects that was a part of the ECoC event. The Foundation has created multiple KPIs on a strategical/political level to assess the success of the event. However, the GPT has focused on making sure that the projects are completed by offering their assistance to the 
projects in the accounting process rather than obsessively focusing on the accomplishment of the KPIs that has been established on a strategical level. The high level of uncertainty in cultural productions may provide a part of an explanation of the pragmatic approach practiced by the GPT.

Our analysis also shows that the contractual relationship of Aarhus 2017 Foundation to the project managers is one based on trust. They believe in the project managers' abilities, benevolence and integrity both to create projects that meet the artistic objectives outlined and to account for their use of financial resources. Accordingly, they do not, as in conventional thinking of principal-agency theory, perceive the project managers as opportunistic agents, who are motivated to act in the interest of the Aarhus 2017 only if the action is within their self-interest. However, this does not mean that Aarhus 2017 and the GPT employees have blind faith in the project managers. Indeed, they observe and monitor both the cultural content of the projects and the project managers' use of financial resources.

The GPT applies a simple accounting system to detect patterns of deviation. Further, trust is established through an interactive process of questioning and a reflective learning process with the participating projects of the ECoC event in Aarhus. Through the learning process, the actor(s) undergo(es) conceptual development to continuously improve the level of insight and diagnostic certainty to establish a pragmatic truth on trust, enabling the actor(s) to deliver more trustworthy accounting reporting. 